\title{
Optimum Pilot Overhead in Wireless Communication: A Unified Treatment of Continuous and Block-Fading Channels
}

\author{
Angel Lozano \\ Universitat Pompeu Fabra (UPF) \\ Barcelona 08018, Spain \\ Email: angel.lozano@upf.edu
}

\author{
Nihar Jindal \\ University of Minnesota \\ Minneapolis, MN 55455, USA \\ Email: nihar@umn.edu
}

\begin{abstract}
The optimization of the pilot overhead in wireless fading channels is investigated, and the dependence of this overhead on various system parameters of interest (e.g., fading rate, signal-to-noise ratio) is quantified. The achievable pilot-based spectral efficiency is expanded with respect to the fading rate about the no-fading point, which leads to an accurate order expansion for the pilot overhead. This expansion identifies that the pilot overhead, as well as the spectral efficiency penalty with respect to a reference system with genie-aided CSI (channel state information) at the receiver, depend on the square root of the normalized Doppler frequency. It is also shown that the widely-used block fading model is a special case of more accurate continuous fading models in terms of the achievable pilotbased spectral efficiency. Furthermore, it is established that the overhead optimization for multiantenna systems is effectively the same as for single-antenna systems with the normalized Doppler frequency multiplied by the number of transmit antennas.
\end{abstract}

\section{INTRODUCTION}

Most wireless communication systems perform coherent data detection with the assistance of pilot signals (a.k.a. reference signals or training sequences) that are inserted periodically [1]. The receiver typically performs channel estimation on the basis of the received pilot symbols, and then applies standard coherent detection while treating the channel estimate as if it were the true channel. When such an approach is taken ${ }^{1}$ and Gaussian inputs are used, the channel estimation error effectively introduces additional Gaussian noise [3]. This leads to a non-trivial tradeoff: increasing the fraction and/or power of pilot symbols improves the channel estimation quality and thus decreases this additional noise, but also decreases the fraction and/or power of data symbols. To illustrate the importance of this tradeoff, Fig. 1 depicts the spectral efficiency as a function of the pilot overhead (cf. Section III for details) for some standard channel

\footnotetext{
${ }^{1}$ The focus of this paper is on separate processing of pilots and data. Other approaches such as data-aided channel estimation [2] or semiblind estimation are feasible, but are not currently employed and are not investigated here.
}

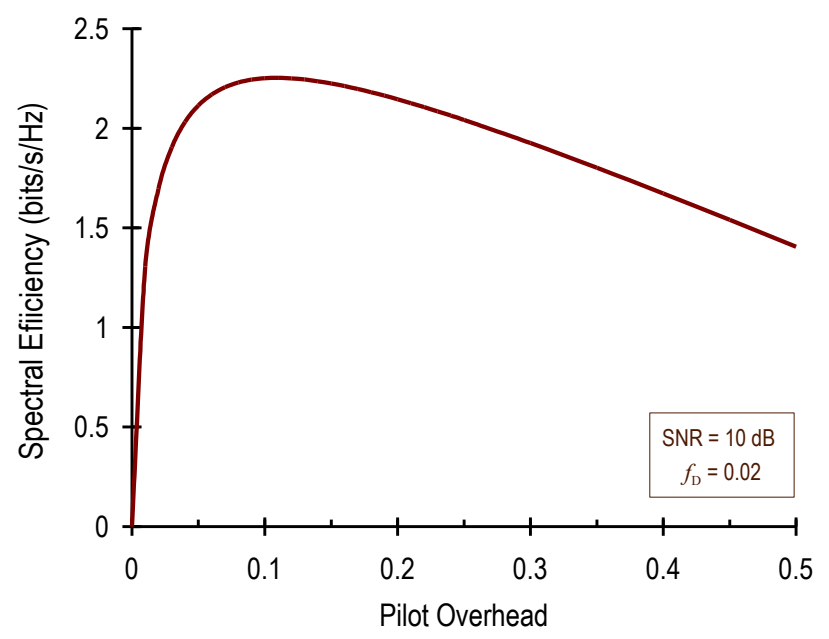

Fig. 1. Spectral efficiency as a function of the pilot overhead for $\mathrm{SNR}=10 \mathrm{~dB}$. The Doppler spectrum is Clarke-Jakes with a maximum normalized frequency $f_{\mathrm{D}}=0.02$ corresponding, for instance, to 100 $\mathrm{Km} / \mathrm{h}$ in a WiMAX system. The pilot and data symbols have equal power.

conditions. Clearly, an incorrect overhead can greatly diminish the achievable spectral efficiency.

Note that Fig. 1 corresponds to a high-velocity setting and thus the spectral efficiency presented therein presumes that the transmit rate is not varied dynamically as a function of the multipath fading, but rather held constant over such fading and adapted only to the average signal-to-noise ratio. This therefore corresponds to the so-called "ergodic" spectral efficiency. At lower velocities, it is possible to perform adaptive rate control on the basis of feedback from the receiver to the transmitter and to thereby match the instantaneous rate to the multipath fading, but as long as the fading is ergodic the temporal average of the achievable rates continues to map to the same ergodic spectral efficiency. Thus, settings with and without adaptive rate control are covered by our analysis.

The aforementioned tradeoff has been extensively 
studied in the literature [3]-[10], on the basis of both simplified block-fading models as well as more accurate continuous-fading models. In most systems, the pilot symbol power is fixed due to peak-to-average and interference considerations and thus the optimization is only over the fraction of pilot symbols. This version of the optimization has been posed in prior work [5], [7][9], and explicit results have been established in the lowand high-power asymptotes. However, these asymptotes become accurate only for extreme power levels [10]; as a result, this version of the optimization must generally be solved numerically. In the case that both the power and fraction of pilot symbols can be varied, a closed-form solution for the optimal power and fraction is known for the special cases of block-fading [5] and continuousfading with a rectangular Doppler spectrum [8], [9].

In this paper, we study the overhead optimization (with and without pilot power boosting) in the limiting regime of slow fading. More precisely, by expanding the spectral efficiency around the perfect-CSI point, i.e., for small fading rates, both versions of the optimizations can be tackled and useful expansions (in terms of the fading rate) for the optimum pilot overhead are obtained. These expansions are seen to be accurate over a wide range of relevant operating points, and provide valuable insight on the dependence of the optimal overhead and corresponding spectral efficiency on the various parameters of interest (velocity, power, etc). For non-boosted pilots in particular, these expansions are the first explicit expressions that capture these dependencies for arbitrary power levels.

Some additional insights reached in the paper are:

- In terms of the spectral efficiency achievable with pilot-based communication, block-fading is a special case of continuous (symbol-by-symbol) fading.

- The optimal pilot overhead scales with the square root of the Doppler frequency; this result holds regardless of whether pilot power boosting is allowed.

- The spectral efficiency penalty w.r.t. the perfectCSI capacity also scales with the square-root of the fading rate.

- The pilot overhead optimization for multiantenna transmission is essentially the same as the optimization for single-antenna transmission except with the true Doppler frequency multiplied by the number of transmit antennas. If the Doppler is fixed, therefore, the overhead scales with the square root of the number of transmit antennas regardless of whether pilot power boosting is allowed.

By showing that block-fading is a special case of the richer set of continuous fading models, the two models are unified in the context of pilot-based communication. This is important because, thus far, these models had been treated separately (block fading is used in [4]-[6] while continuous-fading is considered in [7]-[10]) and no connection had been established between them. In this paper we show that the two need not be separately treated; moreover, results obtained for one model can be applied to the other. The connection established between single-antenna and multiantenna transmission has similar benefits.

\section{Preliminaries}

\section{A. Channel Model}

Consider a discrete-time frequency-flat scalar fading channel $H(k)$ where $k$ is the time index. (The extension to multiantenna channels is considered in Section VI.) Pilot symbols are inserted periodically in the transmission and the fraction thereof is denoted by $\alpha$, i.e., one in every $1 / \alpha$ symbols is a pilot while the rest are data. ${ }^{2}$ Moreover, $\alpha \geq \alpha_{\min }$ where $\alpha_{\min }$ is established later in this section.

Let $\mathcal{D}$ denote the set of time indices corresponding to data symbols. For $k \in \mathcal{D}$,

$$
Y(k)=H(k) \sqrt{P} X(k)+N(k)
$$

where the transmitted signal, $X(k)$, is a sequence of IID (independent identically distributed) complex Gaussian random variables with zero mean and unit variance that we indicate by $X \sim \mathcal{N}_{\mathbb{C}}(0,1)$. The additive noise is $N \sim$ $\mathcal{N}_{\mathbb{C}}\left(0, N_{0}\right)$ and we define SNR $=P / N_{0}$.

For $k \notin \mathcal{D}$, unit-amplitude pilots are transmitted and thus

$$
Y(k)=H(k) \sqrt{P}+N(k) .
$$

Notice that pilot symbols and data symbols have the same average power. In Section V, we shall lift this constraint allowing for power-boosted pilots.

1) Block Fading: In the popular block-fading model, the channel is drawn as $H \sim \mathcal{N}_{\mathbb{C}}(0,1)$ at the beginning of each block and it then remains constant for the $n_{\mathrm{b}}$ symbols composing the block. This process is repeated for every block in an IID fashion.

In order for the receiver to estimate the channel, at least one pilot symbol must be inserted within each block. If $n_{\mathrm{p}}$ represents the number of pilot symbols in every block, then

$$
\alpha=\frac{n_{\mathrm{p}}}{n_{\mathrm{b}}}
$$

and clearly $\alpha_{\min }=1 / n_{\mathrm{b}}$.

2) Continuous Fading: In this model, $H(k)$ is a discretetime complex Gaussian stationary random process, with an absolutely continuous spectral distribution function whose derivative is the Doppler spectrum $S_{H}(\nu)$, $-1 / 2 \leq \nu \leq 1 / 2$ [11]. It follows that the channel is ergodic.

\footnotetext{
${ }^{2}$ Although $\alpha$ should be restricted to integer-reciprocals, our derivations relax this constraint. Thus, our results should be rounded to an integer-reciprocal.
} 
We consider bandlimited processes such that

$$
\begin{cases}S_{H}(\nu)>0, & |\nu| \leq f_{\mathrm{D}} \\ S_{H}(\nu)=0, & |\nu|>f_{\mathrm{D}}\end{cases}
$$

for some Doppler frequency $f_{\mathrm{D}} \leq 1 / 2$. The Doppler frequency is typically given by $f_{\mathrm{D}}=T v / \lambda$, where $T$ is the symbol period, $v$ is the velocity, and $\lambda$ is the carrier wavelength.

To ensure that the decimated channel observed through the pilot transmissions has an unaliased spectrum, it is necessary that

$$
\alpha_{\min }=2 f_{\mathrm{D}} .
$$

On account of its bandlimited nature, the channel is a nonregular fading process [11]. We further consider $S_{H}(\cdot)$ to be strictly positive within $\pm f_{\mathrm{D}} \cdot{ }^{3}$ In order to remain consistent with earlier definitions of signal and noise power, only unit-power processes are considered.

Two important spectra are the Clarke-Jakes [12]

$$
S_{H}(\nu)=\frac{1}{\pi \sqrt{f_{\mathrm{D}}^{2}-\nu^{2}}}
$$

and the rectangular

$$
S_{H}(\nu)= \begin{cases}1 /\left(2 f_{\mathrm{D}}\right) & |\nu| \leq f_{\mathrm{D}} \\ 0 & |\nu|>f_{\mathrm{D}}\end{cases}
$$

We will later find it useful to express the Doppler spectrum as

$$
S_{H}(\nu)=\frac{1}{f_{\mathrm{D}}} \tilde{S}_{H}\left(\frac{\nu}{f_{\mathrm{D}}}\right)
$$

where $\tilde{S}_{H}(\cdot)$ is a normalized spectral shape bandlimited to \pm 1 . For the Clarke-Jakes spectrum in (6), for instance, the spectral shape is

$$
\tilde{S}_{H}(\nu)=\frac{1}{\pi \sqrt{1-\nu^{2}}}
$$

while, for the rectangular spectrum in (7), the spectral shape is

$$
\tilde{S}_{H}(\nu)= \begin{cases}1 / 2 & |\nu| \leq 1 \\ 0 & |\nu|>1\end{cases}
$$

\section{B. Perfect CSI}

With perfect CSI at the receiver, i.e., assuming a genie provides the receiver with $H(k)$, there is no need for pilot symbols $(\alpha=0)$. The capacity in bits $/ \mathrm{s} / \mathrm{Hz}$ is then [13]

$$
\begin{aligned}
C(\mathrm{SNR}) & =\mathbb{E}\left[\log _{2}\left(1+\mathrm{SNR}|H|^{2}\right)\right] \\
& =\log _{2}(e) e^{1 / \mathrm{SNR}} E_{1}\left(\frac{1}{\mathrm{SNR}}\right)
\end{aligned}
$$

\footnotetext{
${ }^{3}$ This premise can be easily removed by simply restricting all the integrals in the paper to the set of frequencies where $S_{H}(\nu)>0$, rather than to the entire interval $\pm f_{\mathrm{D}}$.
}

where $E_{1}(\zeta)=\int_{1}^{\infty} t^{-1} e^{-\zeta t} d t$ is the exponential integral. The first derivative of $C(\cdot)$ can be conveniently expressed as a function of $C(\cdot)$ via

$$
\dot{C}(\mathrm{SNR})=\frac{1}{\mathrm{SNR}}\left(\log _{2} e-\frac{C(\mathrm{SNR})}{\mathrm{SNR}}\right) .
$$

In turn, the second derivative can be expressed as a function of $C(\cdot)$ and $\dot{C}(\cdot)$ as

$$
\ddot{C}(\mathrm{SNR})=-\frac{1}{\mathrm{SNR}^{2}}\left[\log _{2} e+\dot{C}(\mathrm{SNR})-2 \frac{C(\mathrm{SNR})}{\mathrm{SNR}}\right] .
$$

\section{Pilot-Assisted Detection}

In pilot-assisted communication, decoding must be conducted on the basis of the channel outputs (data and pilots) alone, without the assistance of genie-provided channel realizations. In this case, the maximum spectral efficiency that can be achieved reliably is the mutual information between the data inputs and the outputs (data and pilots). This mutual information equals

$$
\lim _{K \rightarrow \infty} \frac{1}{K} I(\underbrace{\{X(k)\}_{k=0}^{K-1} ;\{Y(k)\}_{k=0}^{K-1}}_{k \in \mathcal{D}} \mid \underbrace{\{Y(k)\}_{k=0}^{K-1}}_{k \notin \mathcal{D}})
$$

where $K$ is the coding blocklength in symbols. Achieving (15), for which there is no known simplified expression, generally requires joint data decoding and channel estimation.

Contemporary wireless systems take the lower complexity, albeit suboptimal, approach of first estimating the channel for each data symbol-based exclusively upon all received pilot symbols - and then performing nearest-neighbor decoding using these channel estimates as if they were correct. This is an instance of mismatched decoding. If we express the channel as $H(k)=\hat{H}(k)+$ $\tilde{H}(k)$ where $\hat{H}(k)$ denotes the minimum mean-square error estimate of $H(k)$, the received symbol can be rewritten as

$$
Y(k)=\hat{H}(k) \sqrt{P} X(k)+\tilde{H}(k) \sqrt{P} X(k)+N(k) .
$$

Performing nearest-neighbor decoding as described above $^{4}$ has been shown to have the effect of making the term $\tilde{H}(k) \sqrt{P} X(k)$ appear as an additional source of independent Gaussian noise [14]. With that, the spectral efficiency becomes [3]-[5], [7], [8]

$$
\bar{I}(\mathrm{SNR}, \alpha)=(1-\alpha) C\left(\mathrm{SNR}_{\mathrm{eff}}\right)
$$

with

$$
\mathrm{SNR}_{\mathrm{eff}}=\frac{\mathrm{SNR}(1-\mathrm{MMSE})}{1+\mathrm{SNR} \cdot \mathrm{MMSE}}
$$

where MMSE $=\mathbb{E}\left[|\tilde{H}|^{2}\right]$. Although not explicitly indi-

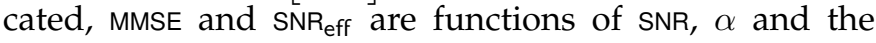
underlying fading model.

\footnotetext{
${ }^{4}$ More specifically, the decoder finds the codeword $[X(1), \ldots, X(K)]$ that minimizes the distance metric $\sum_{k=1}^{K}|Y(k)-\sqrt{P} \hat{H}(k) X(k)|^{2}$.
} 
In addition to representing the maximum spectral efficiency achievable with Gaussian codebooks and channelestimate-based nearest-neighbor decoding, $\bar{I}(\cdot)$ is also a lower bound to (15). Because of this double significance, the maximization of $\bar{I}(\cdot)$ over $\alpha$

$$
\bar{I}^{\star}(\mathrm{SNR})=\max _{\alpha_{\min } \leq \alpha \leq 1} \bar{I}(\mathrm{SNR}, \alpha)
$$

and especially the argument of such maximization, $\alpha^{\star}$, are the focal points of this paper.

The expressions in (17) and (19) apply to both block and continuous fading, and these settings differ only in how MMSE behaves as a function of $\alpha$ and SNR.

In block fading, $n_{\mathrm{p}}$ pilot symbols are used to estimate the channel in each block and thus [5]

$$
\text { MMSE }=\frac{1}{1+\alpha n_{\mathrm{b}} \mathrm{SNR}} .
$$

For continuous fading, on the other hand [1], [8]

$$
\begin{aligned}
\text { MMSE } & =1-\int_{-f_{\mathrm{D}}}^{+f_{\mathrm{D}}} \frac{\operatorname{SNR} S_{H}^{2}(\nu)}{1 / \alpha+\operatorname{SNR} S_{H}(\nu)} d \nu \\
& =1-\int_{-1}^{+1} \frac{\tilde{S}_{H}^{2}(\xi)}{\frac{f_{\mathrm{D}}}{\alpha \mathrm{SNR}}+\tilde{S}_{H}(\xi)} d \xi
\end{aligned}
$$

where (22) is based upon the spectral shape definition in (8).

For the Clarke-Jakes spectrum, MMSE can be computed in closed-form as [10]

$$
\text { MMSE }=1-\frac{\operatorname{arctanh} \sqrt{1-\left(\frac{\alpha \mathrm{SNR}}{\pi f_{\mathrm{D}}}\right)^{2}}}{\frac{\pi}{2} \sqrt{\left(\frac{\pi f_{\mathrm{D}}}{\alpha \mathrm{SNR}}\right)^{2}-1}}
$$

while, for the rectangular spectrum [8]

$$
\text { MMSE }=\frac{1}{1+\frac{\alpha}{2 f_{\mathrm{D}}} \mathrm{SNR}} .
$$

Proposition 1 For any pilot overhead $\alpha$, the spectral efficiency achievable with pilot-based communication, i.e., $\bar{I}(\mathrm{SNR}, \alpha)$, on a block-fading channel with blocksize $n_{\mathrm{b}}$ equals the spectral efficiency achievable on a continuous-fading channel with a rectangular spectrum where

$$
f_{\mathrm{D}}=\frac{1}{2 n_{\mathrm{b}}} .
$$

Proof: Comparing (20) with (24), the block-fading model is seen to yield the same MMSE as a continuous fading model with a rectangular spectrum if $f_{\mathrm{D}}=1 /\left(2 n_{\mathrm{b}}\right)$. Because $\bar{I}(\cdot)$ depends on the fading model only through MMSE, this implies equivalence in terms of spectral efficiency.

Thus, for the remainder of the paper we consider continuous fading keeping in mind that block-fading corresponds to the special case of a rectangular spectrum with (25).
Notice by applying Jensen's inequality to the integral in (22), using the fact that the function $x^{2} /(a+x)$ is convex in $x$ for any $a>0$, that the MMSE for any spectrum (and fixed $f_{\mathrm{D}}$ ) is lower bounded by the right-hand side of (24). In other words, the rectangular spectrum results in the worst case estimation error; thus, block-fading analyses are also worst-case.

\section{Pilot Overhead Optimization}

The optimization in (19) does not yield an explicit solution, even for the simplest of fading models, and therefore it must be computed numerically. In this section, we circumvent this difficulty by appropriately expanding the objective function $\bar{I}(\cdot)$. This leads to a simple expression that cleanly illustrates the dependence of $\alpha^{*}$ and $\bar{I}^{*}$ on the parameters of interest.

In particular, we shall expand (17) with respect to $f_{\mathrm{D}}$ while keeping the shape of the Doppler spectrum fixed (but arbitrary). Besides being analytically convenient, this approach correctly models different velocities within a given propagation environment. ${ }^{5}$ We shall henceforth explicitly indicate the dependence of $\bar{I}(\cdot)$ and $\bar{I}^{\star}(\cdot)$ on $f_{\mathrm{D}}$.

Proposition 2 The optimum pilot overhead for a Rayleighfaded channel with an arbitrary bandlimited Doppler spectrum expands as

$$
\begin{aligned}
\alpha^{\star}= & \sqrt{(1+\mathrm{SNR}) \frac{\dot{C}(\mathrm{SNR})}{C(\mathrm{SNR})} 2 f_{\mathrm{D}}} \\
& -\left((1+\mathrm{SNR}) \frac{\ddot{C}(\mathrm{SNR})}{\dot{C}(\mathrm{SNR})}+2+\frac{1}{2 \mathrm{SNR}} \int_{-1}^{+1} \frac{d \xi}{\tilde{S}_{H}(\xi)}\right) f_{\mathrm{D}} \\
& +O\left(f_{\mathrm{D}}^{3 / 2}\right)
\end{aligned}
$$

where $\tilde{S}_{H}(\nu)$ is the spectral shape defined in (8). The corresponding spectral efficiency expands as

$$
\begin{aligned}
\bar{I}^{\star}\left(\mathrm{SNR}, f_{\mathrm{D}}\right)= & C(\mathrm{SNR})-\sqrt{8 f_{\mathrm{D}}(1+\mathrm{SNR}) C(\mathrm{SNR}) \dot{C}(\mathrm{SNR})} \\
& +O\left(f_{\mathrm{D}}\right)
\end{aligned}
$$

Proof: See [16].

The expression for $\alpha^{\star}$ in Proposition 2 is a simple function involving the perfect-CSI capacity and its derivatives (cf. Section II). Furthermore, the leading term in the expansion does not depend on the particular spectral shape. Only the subsequent term begins to exhibit such dependence, through

$$
\int_{-1}^{+1} d \nu / \tilde{S}_{H}(\nu)
$$

For a Clarke-Jakes spectrum, for instance, this integral equals $\pi^{2} / 2$. For a rectangular spectrum, it equals 4 .

\footnotetext{
${ }^{5}$ The propagation environment determines the spectral shape while the velocity determines $f_{\mathrm{D}}$.
} 


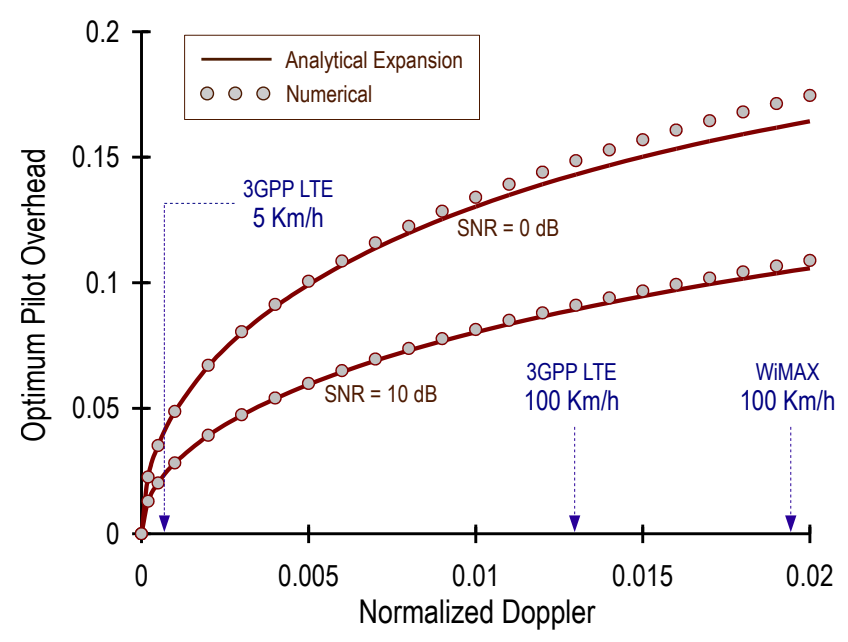

Fig. 2. Optimum pilot overhead, $\alpha^{\star}$, as a function of $f_{\mathrm{D}}$ for $\mathrm{SNR}=0$ $\mathrm{dB}$ and $\mathrm{SNR}=10 \mathrm{~dB}$ with a Clarke-Jakes spectrum. Relevant Doppler levels for LTE and WiMAX are highlighted.

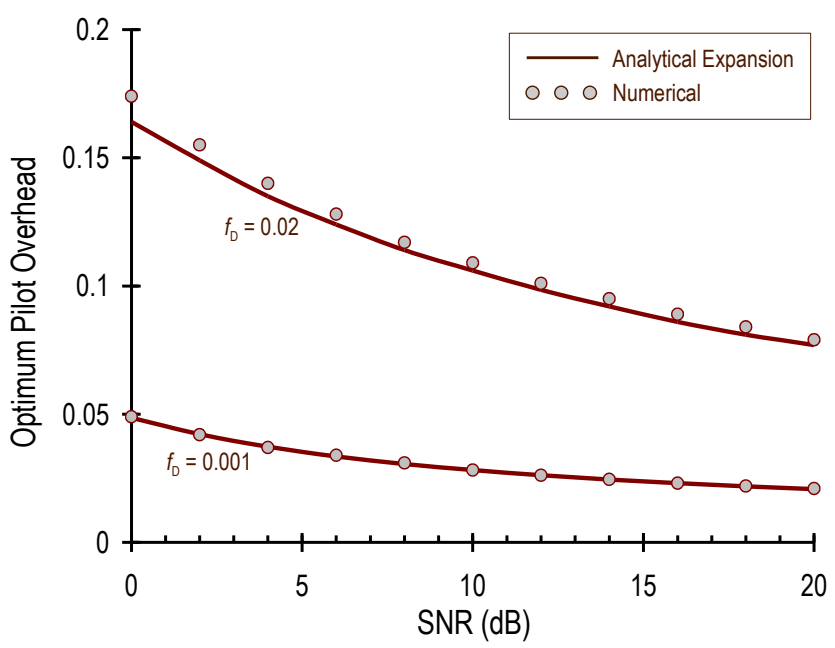

Fig. 3. Optimum pilot overhead, $\alpha^{\star}$, as a function of SNR for $f_{\mathrm{D}}=$ 0.001 and $f_{\mathrm{D}}=0.02$ with a Clarke-Jakes spectrum.

Comparisons between the optimum pilot overhead expansion in Proposition 2 and the corresponding exact value obtained numerically are presented in Figs. 23. The agreement is excellent for essentially the entire range of Doppler and SNR values of interest in mobile wireless systems. As with the optimum overhead, good agreement is shown in Figs. 4-5 between the spectral efficiency in (27) and its numerical counterpart as rendered by (19).

A direct insight of Proposition 2 is that the optimum pilot overhead, $\alpha^{*}$, and the spectral efficiency penalty w.r.t. the perfect-CSI capacity, $C(\mathrm{SNR})-\bar{I}^{\star}\left(\mathrm{SNR}, f_{\mathrm{D}}\right)$, both depend on the Doppler as $\sqrt{f_{\mathrm{D}}}$. To gain an intuitive understanding of this scaling, we can express such penalty

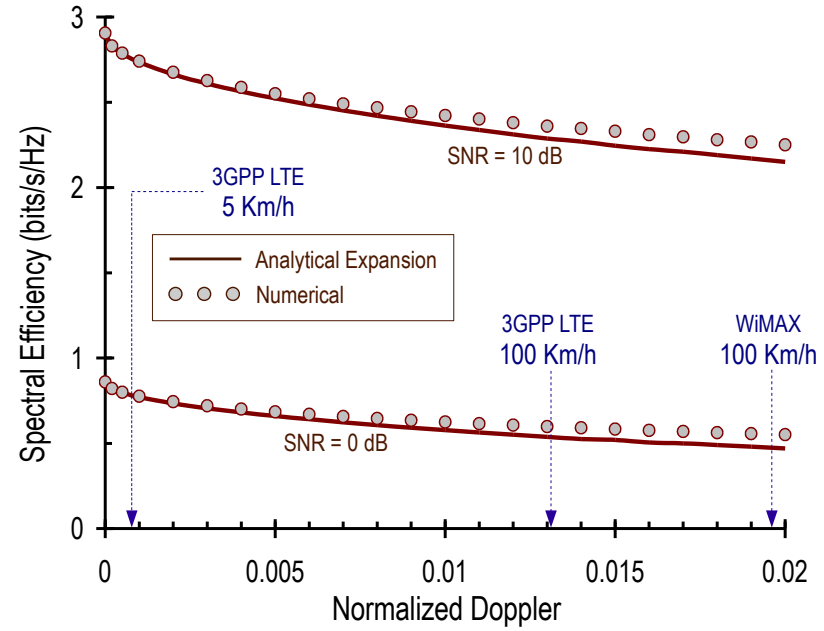

Fig. 4. Spectral efficiency with optimum pilot overhead as a function of $f_{\mathrm{D}}$ for $\mathrm{SNR}=10 \mathrm{~dB}$ with a Clarke-Jakes spectrum. Relevant normalized Doppler levels for LTE and WiMAX are highlighted.

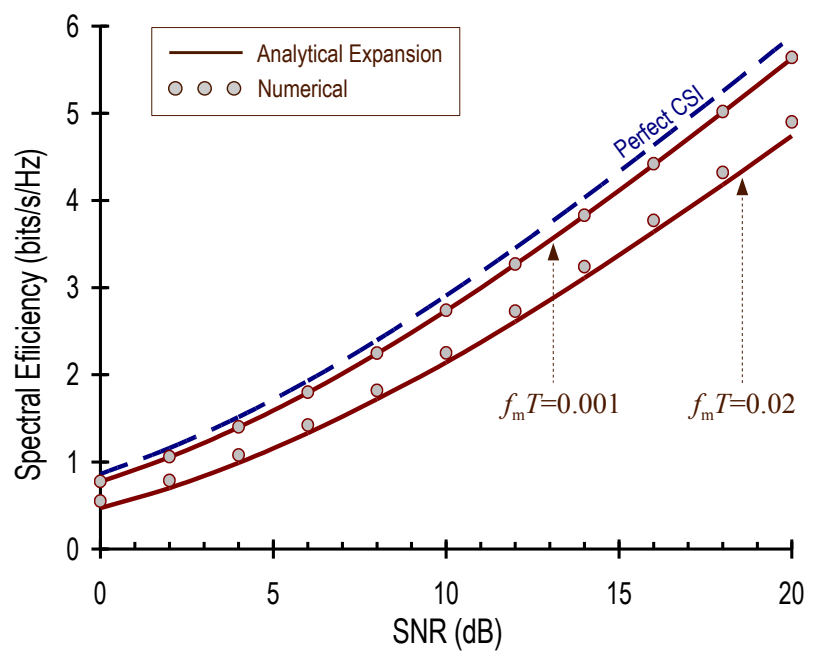

Fig. 5. Spectral efficiency with optimum pilot overhead as a function of SNR for $f_{\mathrm{D}}=0.001$ and $f_{\mathrm{D}}=0.02$ with a Clarke-Jakes spectrum. Also shown is the capacity with perfect CSI.

for an arbitrary $\alpha$ as [16, Appendix A]

$$
\begin{aligned}
C(\mathrm{SNR})-\bar{I}\left(\mathrm{SNR}, \alpha, f_{\mathrm{D}}\right)= & \alpha C(\mathrm{SNR})+\frac{(1+\mathrm{SNR}) \dot{C}(\mathrm{SNR}) 2 f_{\mathrm{D}}}{\alpha} \\
& +O\left(f_{\mathrm{D}}\right) .
\end{aligned}
$$

The first term in (29) represents the spectral efficiency loss because only a fraction $(1-\alpha)$ of the symbols contain data, while the second term is the loss on those transmitted data symbols due to the inaccurate CSI. If $\alpha$ is chosen to be $O\left(f_{\mathrm{D}}^{s}\right)$ for $0 \leq s \leq 1$, the first and second terms in (29) are $O\left(f_{\mathrm{D}}^{s}\right)$ and $O\left(f_{\mathrm{D}}^{1-s}\right)$, respectively, and thus the overall penalty is

$$
O\left(f_{\mathrm{D}}^{\min \{s, 1-s\}}\right) .
$$


Hence, the spectral efficiency penalty is minimized by balancing the two terms and selecting $\alpha^{\star}=O\left(\sqrt{f_{\mathrm{D}}}\right)$.

It is important to realize that, although the expansion in Proposition 2 is remarkably accurate for a wide range of SNR values, it becomes less accurate for SNR $\rightarrow 0$ or SNR $\rightarrow \infty$. In fact, in limiting SNR regimes it is possible to explicitly handle arbitrary Doppler levels [4], [5], [9], [10]. Thus, it is precisely for intermediate SNR values where the analysis here is both most accurate and most useful, thereby complementing those in the aforegiven references.

\section{Pilot Power Boosting}

In some systems, it is possible to allocate unequal powers for pilot and data symbols. In our models, this can be accommodated by defining the signal-to-noise ratios for pilot and data symbols to be $\rho_{\mathrm{p}} \mathrm{SNR}$ and $\rho_{\mathrm{d}} \mathrm{SNR}$, respectively, with

$$
\rho_{\mathrm{p}} \alpha+\rho_{\mathrm{d}}(1-\alpha)=1
$$

so that the average transmitted power is preserved. The spectral efficiency in (17) continues to hold, only with

$$
\mathrm{SNR}_{\mathrm{eff}}=\frac{\mathrm{SNR}(1-\mathrm{MMSE})}{1 / \rho_{\mathrm{d}}+\mathrm{SNR} \cdot \mathrm{MMSE}} .
$$

The expressions for MMSE in (20) and (21) hold with SNR replaced with $\rho_{\mathrm{p}}$ SNR. As a result, with block fading,

$$
\text { MMSE }=\frac{1}{1+\alpha n_{\mathrm{b}} \rho_{\mathrm{p}} \mathrm{SNR}}
$$

while, with continuous fading,

$$
\text { MMSE }=1-\int_{-f_{\mathrm{D}}}^{+f_{\mathrm{D}}} \frac{\mathrm{SNR} S_{H}^{2}(\nu)}{1 /\left(\rho_{\mathrm{p}} \alpha\right)+\operatorname{sNR} S_{H}(\nu)} d \nu
$$

It is easily verified, from (33) and (34), that the identity between block fading and continuous fading with a rectangular Doppler spectrum continues to hold under condition (25).

It can be inferred, from (17), (32) and (34), that it is advantageous to increase $\rho_{\mathrm{p}}$ while simultaneously reducing $\alpha$ all the way to $\alpha_{\min }$. Indeed, for the block-fading model, the observation is made in [4], [5] that, with pilot power boosting, a single pilot symbol should be inserted on every fading block. With continuous fading, that translates to

$$
\alpha=2 f_{\mathrm{D}}
$$

and the issue is then the optimization of $\rho_{\mathrm{p}}$ and $\rho_{\mathrm{d}}$. Because $\alpha$ is fixed, the power boosting that maximizes $\bar{I}(\cdot)$ is directly the one that maximizes $S N R_{\text {eff, }}$, i.e.,

$$
\begin{aligned}
\rho_{\mathrm{p}}^{\star} & =\arg \max _{\rho_{\mathrm{p}} \alpha_{\min }+\rho_{\mathrm{d}}\left(1-\alpha_{\min }\right)=1} \bar{I}(\mathrm{SNR}, \alpha) \\
& =\arg \max _{\rho_{\mathrm{p}} \alpha_{\min }+\rho_{\mathrm{d}}\left(1-\alpha_{\text {min }}\right)=1} \text { SNR }_{\text {eff }} .
\end{aligned}
$$

Although simpler than the optimization in Section IV, this nonetheless must be computed numerically with the

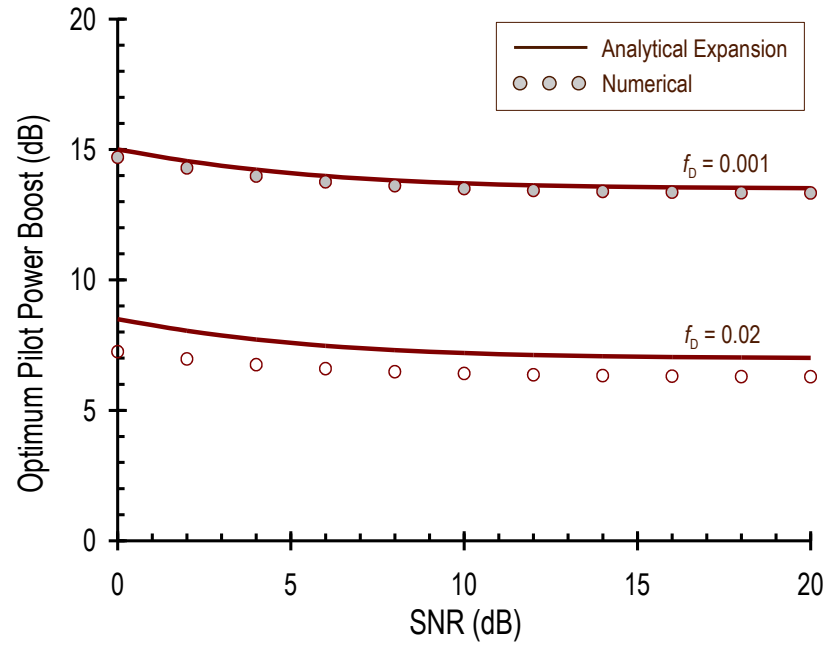

Fig. 6. Optimum pilot power boost, $\rho_{\mathrm{p}}^{\star}$, as a function of SNR for $f_{\mathrm{D}}=0.001$ and $f_{\mathrm{D}}=0.02$ with a Clarke-Jakes spectrum.

exception of the rectangular spectrum/block-fading [5, Thm 2].

As in Section III, we circumvent this limitation by expanding the problem in $f_{\mathrm{D}}$. Again, this yields expressions that are explicit and valid for arbitrary spectral shapes.

Proposition 3 The optimum power allocation for a Rayleighfaded channel with an arbitrary bandlimited Doppler spectrum is given by

$$
\begin{aligned}
& \rho_{\mathrm{p}}^{\star}=\sqrt{\frac{1+1 / \mathrm{SNR}}{2 f_{\mathrm{D}}}}+O(1) \\
& \rho_{\mathrm{d}}^{\star}=1-\sqrt{\left(1+\frac{1}{\mathrm{SNR}}\right) 2 f_{\mathrm{D}}}+O\left(f_{\mathrm{D}}\right)
\end{aligned}
$$

and the corresponding spectral efficiency is

$\bar{I}^{\star}\left(\mathrm{SNR}, f_{\mathrm{D}}\right)=C(\mathrm{SNR})-\sqrt{8 f_{\mathrm{D}} \mathrm{SNR}(1+\mathrm{SNR})} \dot{C}(\mathrm{SNR})+O\left(f_{\mathrm{D}}\right)$.

Proof: See [16].

As expected, an order expansion of the closed-form solution for the rectangular spectrum [5, Thm 2] matches the above proposition.

A comparison between the optimum pilot power boost given by (38) and the corresponding value obtained numerically is presented in Fig. 6. The agreement is excellent. Good agreement is further shown in Figs. 78 between the corresponding spectral efficiency in (40) and its exact counterpart, again obtained numerically.

\section{Multiantenna Channels}

The analysis extends to multiantenna settings in a straightforward manner when there is no antenna correlation. Letting $n_{\mathrm{T}}$ and $n_{\mathrm{R}}$ denote the number of transmit 


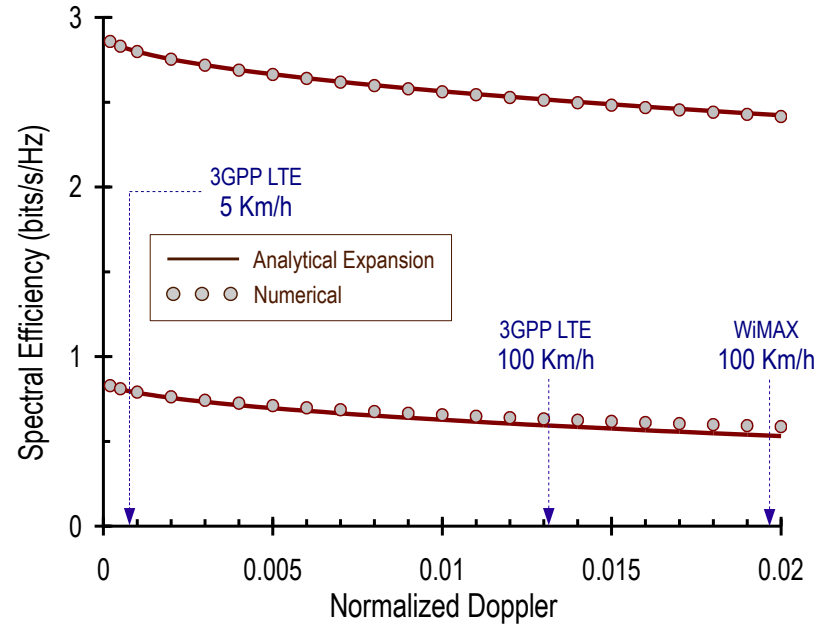

Fig. 7. Spectral efficiency with optimum pilot power boost as a function of $f_{\mathrm{D}}$ for $\mathrm{SNR}=10 \mathrm{~dB}$ with a Clarke-Jakes spectrum. Relevant normalized Doppler levels for LTE and WiMAX are highlighted.

and receive antennas, respectively, the channel at time $k$ is now denoted by the $n_{\mathrm{R}} \times n_{\mathrm{T}}$ matrix $\mathbf{H}(k)$. Each of the $n_{\mathrm{T}} n_{\mathrm{R}}$ entries of the matrix varies in time according to the random processes described in Section II, for either block or continuous fading; furthermore, these processes are independent. Due to such independence, the matrix entries can be separately estimated without loss of optimality. Furthermore, the estimation error is minimized by transmitting orthogonal pilot sequences from the various transmit antennas [5], e.g., transmitting a pilot symbol from a single antenna at a time.

We denote the perfect-CSI multiantenna capacity as

$$
C_{n_{\mathrm{T}}, n_{\mathrm{R}}}(\mathrm{SNR})=\mathbb{E}\left[\log _{2} \operatorname{det}\left(\mathbf{I}+\frac{\mathrm{SNR}}{n_{\mathrm{T}}} \mathbf{H H}^{\dagger}\right)\right],
$$

for which a closed-form expression in terms of the exponential integral can be found in [15].

The spectral efficiency with pilot-assisted detection now becomes [5]

$$
\bar{I}(\mathrm{SNR}, \alpha)=(1-\alpha) C_{n_{\mathrm{T}}, n_{\mathrm{R}}}\left(\mathrm{SNR}_{\mathrm{eff}}\right)
$$

with

$$
\mathrm{SNR}_{\text {eff }}=\frac{\mathrm{SNR}(1-\text { MMSE })}{1+\mathrm{SNR} \cdot \mathrm{MMSE}}
$$

where MMSE is the estimation error for each entry of the channel matrix $\mathbf{H}$.

Because the definition of SNR $_{\text {eff }}$ carries over, the multiantenna formulation mirrors its single-antenna counterpart with $C_{n_{\mathrm{T}}, n_{\mathrm{R}}}(\cdot)$ replacing $C(\cdot)$ and noting that MMSE now indicates the per-entry estimation error. The equivalence between block and continuous fading holds for each entry in terms of MMSE, and thus the equivalence in (25) extends to this multiantenna setting. Therefore, we again restrict our discussion to continuous fading.

Due to the orthogonal pilots, an overhead of $\alpha$ corresponds to a fraction $\alpha / n_{\mathrm{T}}$ of symbols serving as pilots

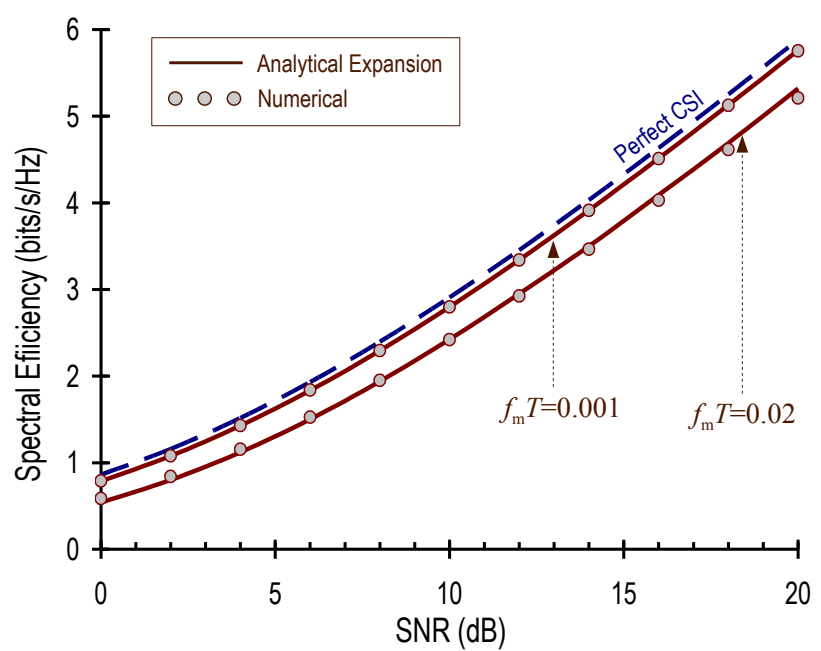

Fig. 8. Spectral efficiency with optimum pilot power boost as a function of SNR for $f_{\mathrm{D}}=0.001$ and $f_{\mathrm{D}}=0.02$ with a Clarke-Jakes spectrum. Also shown is the capacity with perfect CSI.

for a particular transmit antenna (i.e., for the $n_{\mathrm{R}}$ matrix entries associated with that transmit antenna). As a result, the per-entry MMSE is the same as the singleantenna expression in (22) only with $\alpha$ replaced by $\alpha / n_{\mathrm{T}}$, i.e.,

$$
\text { MMSE }=1-\int_{-1}^{+1} \frac{\tilde{S}_{H}^{2}(\xi)}{\frac{n_{\mathrm{T}} f_{\mathrm{D}}}{\alpha \mathrm{SNR}}+\tilde{S}_{H}(\xi)} d \xi .
$$

This equals the MMSE for a single-antenna channel with a Doppler frequency of $n_{\mathrm{T}} f_{\mathrm{D}}$. The optimization w.r.t. $\alpha$ in a multiantenna channel is thus the same as in a single-antenna channel, only with an effective Doppler frequency of $n_{\mathrm{T}} f_{\mathrm{D}}$ and with $C(\cdot)$ replaced by $C_{n_{\mathrm{T}}, n_{\mathrm{R}}}(\cdot)$. As a result, Proposition 2 naturally extends into

$$
\begin{aligned}
\alpha^{\star}= & \sqrt{(1+\mathrm{SNR}) \frac{\dot{C}_{n_{\mathrm{T}}, n_{\mathrm{R}}}(\mathrm{SNR})}{C_{n_{\mathrm{T}}, n_{\mathrm{R}}}(\mathrm{SNR})} 2 n_{\mathrm{T}} f_{\mathrm{D}}} \\
& -\left((1+\mathrm{SNR}) \frac{\ddot{C}_{n_{\mathrm{T}}, n_{\mathrm{R}}}(\mathrm{SNR})}{\dot{C}_{n_{\mathrm{T}}, n_{\mathrm{R}}}(\mathrm{SNR})}+2+\frac{0.5}{\mathrm{SNR}} \int_{-1}^{+1} \frac{d \xi}{\tilde{S}_{H}(\xi)}\right) n_{\mathrm{T}} f_{\mathrm{D}} \\
& +O\left(f_{\mathrm{D}}^{3 / 2}\right) .
\end{aligned}
$$

Notice here the dependence on $\sqrt{n_{\mathrm{T}}}$ in the leading term.

When pilot power boosting is allowed, it is again advantageous to reduce $\alpha$ to its minimum value, now given by $\alpha_{\text {min }}=2 n_{\mathrm{T}} f_{\mathrm{D}}$, and to increase $\rho_{\mathrm{p}}$. In this case the achievable spectral efficiency becomes [5]

$$
\left(1-2 n_{\mathrm{T}} f_{\mathrm{D}}\right) C_{n_{\mathrm{T}}, n_{\mathrm{R}}}\left(\mathrm{SNR}_{\text {eff }}\right)
$$

with SNR $_{\text {eff }}$ as defined in (18) and with

$$
\text { MMSE }=1-\int_{-1}^{+1} \frac{\tilde{S}_{H}^{2}(\xi)}{\frac{n_{\mathrm{T}} f_{\mathrm{D}}}{\alpha \rho_{\mathrm{p}} \mathrm{SNR}}+\tilde{S}_{H}(\xi)} d \xi .
$$

The optimization of the power boost again corresponds to the maximization of $\mathrm{SNR}_{\text {eff }}$ with respect to $\rho_{\mathrm{p}}$. Since 
MMSE is the same as for a single-antenna channel with effective Doppler $n_{\mathrm{T}} f_{\mathrm{D}}$, the optimum pilot power boost for a multiantenna channel with Doppler frequency $f_{\mathrm{D}}$ is exactly the same as the optimum pilot power boost for a single-antenna channel with the same spectral shape and with Doppler frequency $n_{\mathrm{T}} f_{\mathrm{D}}$. As a result, the expressions in Section $\mathrm{V}$ apply verbatim if $f_{\mathrm{D}}$ is replaced by $n_{\mathrm{T}} f_{\mathrm{D}}$.

Based upon these results, when power-boosting is allowed the pilot overhead optimization on a multiantenna channel with Doppler frequency $f_{\mathrm{D}}$ and a particular spectral shape is exactly equivalent to the optimization on a single-antenna channel with the same spectral shape and with Doppler frequency $n_{\mathrm{T}} f_{\mathrm{D}}$. When pilot power boosting is not allowed, this equivalence is approximate because the perfect-CSI capacity functions $C(\cdot)$ and $C_{n_{\mathrm{T}}, n_{\mathrm{R}}}(\cdot)$ differ. Roughly speaking, multiple antennas increase the perfect-CSI capacity by a factor of $\min \left(n_{\mathrm{T}}, n_{\mathrm{R}}\right)$ and thus $C_{n_{\mathrm{T}}, n_{\mathrm{R}}}(\mathrm{SNR}) \approx \min \left(n_{\mathrm{T}}, n_{\mathrm{R}}\right) C(\mathrm{SNR})$. If this approximation were exact, then the aforementioned equivalence would also be exact. Although not exact, the perfect-CSI capacities are sufficiently similar, particularly for symmetric $\left(n_{\mathrm{T}}=n_{\mathrm{R}}\right)$ channels, to render the equivalence very accurate also for the case of nonboosted pilots.

The main implication of the single/multiantenna equivalence is that, based upon our earlier results quantifying the dependence of the pilot overhead on the Doppler frequency, the optimal overhead (with or without power boosting) scales with the number of antennas proportional to $\sqrt{n_{\mathrm{T}}}$.

\section{SUMMARY}

We have investigated the optimization of the pilot overhead in wireless fading channels and quantified the dependence of this overhead on system parameters of interest such as the fading rate and the SNR. Via an expansion with respect to the fading rate about the nofading point, we have identified that the pilot overhead, as well as the spectral efficiency penalty with respect to a reference system with genie-aided CSI at the receiver, depend on the square root of the normalized Doppler frequency. It has also been shown that the widely-used block fading model is a special case of more accurate continuous fading models in terms of the achievable pilot-based spectral efficiency. Furthermore, it has been established that the overhead optimization for multiantenna systems is effectively the same as for singleantenna systems, only with the normalized Doppler frequency multiplied by the number of transmit antennas.

\section{ACKNOWLEDGMENTS}

This research was supported by the government of Spain through the project TEC2009-13000 and the project CONSOLIDER-INGENIO 2010 CSD2008-00010 "COMONSENS".

\section{REFERENCES}

[1] L. Tong, B. M. Sadler, and M. Dong, "Pilot-assisted wireless transmissions: general model, design criteria, and signal processing," IEEE Signal Proc. Magazine, vol. 21, no. 6, pp. 12-25, Nov. 2004.

[2] N. Jindal, A. Lozano, and T. L. Marzetta, "What is the value of joint processing of pilots and data in block-fading channels?," IEEE Int. Symposium on Inform. Theory (ISIT09), Jul. 2009.

[3] M. Medard, "The effect upon channel capacity in wireless communications of perfect and imperfect knowledge of the channel," IEEE Trans. Inform. Theory, vol. 46, no. 3, pp. 933-946, May 2000.

[4] L. Zheng and D. N. C. Tse, "Communication on the Grassman manifold: A geometric approach to the non-coherent multipleantenna channel," IEEE Trans. Inform. Theory, vol. 48, no. 2, pp. 359-383, Feb. 2002.

[5] B. Hassibi and B. M. Hochwald, "How much training is needed in multiple-antenna wireless links?" IEEE Trans. Inform. Theory, vol. 49, no. 4, pp. 951-963, Apr. 2003.

[6] X. Ma, L. Yang, and G. B. Giannakis, "Optimal training for MIMO frequency-selective fading channels," IEEE Trans. Wireless Communications, vol. 4, no. 2, pp. 453-466, Mar. 2005.

[7] J. Baltersee, G. Fock, and H. Meyr, "An information theoretic foundation of synchronized detection," IEEE Trans. Communications, vol. 49, no. 12, pp. 2115-2123, Dec. 2001.

[8] S. Ohno and G. B. Giannakis, "Average-rate optimal PSAM transmissions over time-selective fading channels," IEEE Trans. Wireless Communications, vol. 1, no. 4, pp. 712-720, Oct. 2002.

[9] X. Deng and A. M. Haimovich, "Achievable rates over timevarying Rayleigh fading channels," IEEE Trans. Communications, vol. 55, pp. 1397-1406, Jul. 2007.

[10] A. Lozano, "Interplay of spectral efficiency, power and Doppler spectrum for reference-signal-assisted wireless communication," IEEE Trans. Communications, vol. 56, no. 12, Dec. 2008.

[11] J. Doob, Stochastic Processes. New York, Wiley, 1990.

[12] W. C. Jakes, Microwave Mobile Communications. New York, IEEE Press, 1974.

[13] L. Ozarow, S. Shamai, and A. D. Wyner, "Information theoretic considerations for cellular mobile radio," IEEE Trans. Veh. Technol., vol. 43, pp. 359-378, May 1994.

[14] A. Lapidoth and S. Shamai, "Fading channels: How perfect need 'perfect side information' be?" IEEE Trans. Inform. Theory, vol. 48, pp. 1118-1134, May 2002.

[15] H. Shin and J. H. Lee, "Capacity of multiple-antenna fading channels: Spatial fading correlation, double scattering and keyhole," IEEE Trans. Inform. Theory, vol. 49, pp. 2636-2647, Oct. 2003.

[16] N. Jindal, A. Lozano, "Optimum Pilot Overhead in Wireless Communication: A Unified Treatment of Continuous and BlockFading Channels," arXiv:0903.1379, Mar. 2009. 